\title{
Morphology, clearing efficacy, and mTOR dependency of the organelle autophagoproteasome
}

\author{
Fiona Limanaqi, ${ }^{1 *}$ Francesca Biagioni, ${ }^{2 *}$ Alessandra Salvetti, ${ }^{3}$ Stefano Puglisi-Allegra, ${ }^{2}$ Paola Lenzi, ${ }^{1}$ \\ Francesco Fornai ${ }^{1,2^{*}}$ \\ ${ }^{1}$ Department of Translational Research and New Technologies in Medicine and Surgery, University of Pisa \\ ${ }^{2}$ IRCCS Neuromed, Pozzilli (IS) \\ ${ }^{3}$ Department of Clinical and Experimental Medicine, University of Pisa, Italy \\ *These two authors contributed equally
}

\begin{abstract}
The interplay between autophagy (ATG) and ubiquitin proteasome (UP) cell-clearing systems was recently evidenced at biochemical and morphological levels, where subunits belonging to both pathways co-localize within a novel organelle named autophagoproteasome (APP). We previously documented that APP occurs at baseline conditions, while it is hindered by neurotoxicant administration. This is bound to the activity of the mechanistic target of rapamycin (mTOR), since APP is stimulated by mTOR inhibition, which in turn, is correlated with cell protection. In this brief report, we provide novel morphological and biochemical evidence on APP, suggesting the presence of active UP subunits within ATG vacuoles. Although a stream of interpretation considers such a merging as a catabolic pathway to clear inactive UP subunits, our data further indicate that UP-ATG merging may rather provide an empowered catalytic organelle.
\end{abstract}

Key words: Autophagy; proteasome; LC3, Beclin 1; P20S; alpha-synuclein; transmission electron microscopy; confocal microscopy.

Correspondence: Prof. Francesco Fornai, Department of Translational Research and New Technologies in Medicine and Surgery, University of Pisa, Via Roma 55, 56126 Pisa (PI), and Principal Investigator at IRCCS Neuromed, Via Atinense 18, 86077 Pozzilli (IS), Italy. Tel. +39.050.2218667. E-mail: francesco.fornai@unipi.it, francesco.fornai@neuromed.it

Contributions: FL, writing, review, and editing, data interpretation; FB, cell cultures and immunoprecipitation; AS, confocal microscopy; SPA, data interpretation; PL, transmission electron microscopy; FF, conceptualization and supervision.

Conflict of interest: The authors declare no conflict of interest. 


\section{Introduction}

The eukaryotic cell-clearing systems autophagy (ATG) and ubiquitin proteasome (UP) ensure proteostasis by clearing unfolded, misfolded, oxidized, or disordered proteins. As such, ATG and UP regulate most cell functions including cell cycle and differentiation, immune response, endo- and exocytosis, synaptic plasticity, and neuronal survival. ${ }^{1-15}$ Inhibition of either ATG or UP in experimental models produces detrimental effects which are bound to impaired protein turnover. This is magnified within neuronal cells, which are mostly susceptible to metabolic and oxidative alterations. ${ }^{14,16-25}$ For ages, ATG and UP have been viewed as two independent pathways, concerning different substrate specificity, compartmentalization, and catalytic activity. Challenging such a concept, recent studies documented that a coordinated interplay occurs between the two systems. ${ }^{14,26-29}$ Firstly, several proteins operate at the crossroad between UP and ATG to regulate the sorting and shuttling of protein substrates towards either system, including ubiquitin chains, and the ubiquitin-binding scaffold protein p62. 27,30,31 Again, the mechanistic target of rapamycin (mTOR), which is a well-known ATG-inhibiting kinase, was recently found to modulate the UP. In detail, mTOR inhibition activates overall UP activity aside from it inducing ATG. ${ }^{26}$ Finally, besides operating in the cytosol, UP associates with vesicular organelles, including synaptic vesicles, lysosomes, mitochondria, and ATG-vacuoles. ${ }^{27,28,32}$ Such a placement of free UP within membrane-limited cell compartments underlies a functional cooperation aimed at ensuring the effectiveness of endocytic, secretory/trafficking, and degradation pathways. While vacuolar organelles may shuttle UP back and forth across various cell compartments, ${ }^{32}$ the UP may handle the turnover of vesicle-associated proteins. ${ }^{14}$ This hypothesis follows up recent studies from our research group characterizing the morphology of an organelle named "autophagoproteasome" (APP), where ATG and UP markers (LC3 and P20S, respectively) co-localize at both cellular and ultrastructural levels, as per Autophagy Guidelines policy. $28,29,33$ Again, biochemical experiments documenting the presence of P20S and p62 within LC3-positive immunoprecipitates (roughly corresponding to the APP) suggested molecular binding mechanisms between ATG and UP. Within such LC3 immunoprecipitates hosting P20S we could also detect shared protein substrates, such as alpha-synuclein, likely to be shuttled by p62 as ubiquitinated substrates, along with UP itself, within ATG-vacuoles. ${ }^{27-29}$ The APP occurs at baseline conditions, while it is hindered by the administration of the neurotoxic, synucleinopathy-inducing drug methamphetamine (METH). This is bound to mTOR activity, since APP is stimulated by mTOR inhibition, ${ }^{28}$ which in turn, is correlated with protection against METH toxicity. ${ }^{29}$ This led us to speculate that APP may represent the ultimate cell-clearing organelle, where empowered degradation of potentially harmful, misfolded proteins eventually occurs. As an additional effect, the shuttling of UP within ATG vacuoles is useful to degrade exhausted and inactive UP subunits. ${ }^{27,34}$ In this functional setting, the merging between UP and ATG is defined as "proteaphagy". Whether such a cell compartment represents a system to clear UP or rather it is endowed with empowered catalytic activity, remains to be elucidated. Again, both functions may co-exist. In this report, apart from confirming and extending the morphological characterization of APP at confocal and electron microscopy, we provide evidence on the functional significance of UP merging with ATG.

\section{Materials and Methods}

\section{Cell line}

PC12 cells (IRCCS San Martino Institute, GE, Italy) were grown in RPMI 1640 medium supplemented with heat-inactivated $10 \%$ horse serum and $5 \%$ fetal bovine serum, penicillin $(50$ IU/ml)/streptomycin $(50 \mathrm{mg} / \mathrm{ml}$, Sigma-Aldrich, St. Louis, MO, USA), and maintained in a humidified atmosphere at $5 \% \mathrm{CO}_{2}$ at $37^{\circ} \mathrm{C}$. For transmission electron microscopy (TEM) and immunoprecipitation, $1 \times 10^{6}$ cells were seeded in a final volume of $5 \mathrm{~mL}$. For confocal microscopy, $5 \times 10^{4}$ cells were grown on polylysine slides placed in 24-well plates at a final volume of $1 \mathrm{~mL} /$ well. For confocal microscopy, and immunoprecipitation, cells were treated with the mTOR inhibitor rapamycin $100 \mathrm{nM}$ and $1 \mu \mathrm{M}$ for $72 \mathrm{~h}$ to investigate the effects of mTOR inhibition upon UP activity. Rapamycin doses and timing were chosen from previous studies. $^{28,29}$

\section{Transmission electron microscopy}

Cells were centrifuged at $1000 \mathrm{~g}$ for $5 \mathrm{~min}$, and the pellet was rinsed in PBS and fixed in a solution of $2.0 \%$ paraformaldehyde and $0.1 \%$ glutaraldehyde in $0.1 \mathrm{M}$ PBS ( $\mathrm{pH} 7.4$ ) for 90 min at $4{ }^{\circ} \mathrm{C}$. Specimens were post-fixed in $1 \%$ osmium tetroxide $\left(\mathrm{OsO}_{4}\right)$ for $1 \mathrm{~h}$ at $4{ }^{\circ} \mathrm{C}$, dehydrated in ethanol, and embedded in epoxy resin, as validated in previous studies for immunogold-based ultrastructural morphometry. ${ }^{28,29,35-38}$ Ultrathin sections were collected on nickel grids, and incubated on droplets of aqueous sodium meta-periodate $\left(\mathrm{NaIO}_{4}\right)$, for $30 \mathrm{~min}$, at $22^{\circ} \mathrm{C}$ to remove $\mathrm{OsO}_{4}$. Grids were washed in PBS and incubated in a blocking solution of $10 \%$ goat serum and $0.2 \%$ saponin for $20 \mathrm{~min}$, at room temperature (RT). Grids were then incubated with the primary antibody solution containing rabbit anti-LC3, anti-Beclin1, or anti-alpha-syn (1:50), and mouse anti-P20S (1:50, Abcam, Cambridge, UK), with $0.2 \%$ saponin and $1 \%$ goat serum in a humidified chamber overnight, at $4^{\circ} \mathrm{C}$. After washing in PBS, grids were incubated with the secondary antibodies conjugated with gold particles $(1: 30,10 \mathrm{~nm}$ diameter for antirabbit; $20 \mathrm{~nm}$ diameter for anti-mouse, BB International) in $0.2 \%$ saponin and $1 \%$ goat serum PBS, for $1 \mathrm{~h}$, at RT. After washing in PBS, grids were incubated on $1 \%$ glutaraldehyde droplets for $3 \mathrm{~min}$ and washed in distilled water. Counts of immunogold particles were performed directly at TEM at $8000 \mathrm{x}$ magnification for concomitant identification of immunogold particles and cell organelles. Assessment of APP and measurement of immunogold particles were carried out according to previous studies and the "Guidelines for the Use and Interpretation of Assays for Monitoring Autophagy ( $3^{\text {rd }}$ edition)..$^{28,29,33}$ Samples were examined using a JEOL JEM-100SX TEM (JEOL, Tokyo, Japan).

\section{Confocal Microscopy}

Cells were PBS-washed and fixed with paraformaldehyde 4\% for $5 \mathrm{~min}$ at RT. Antigen retrieval was carried out in $100 \mathrm{mM}$ Tris$\mathrm{HCl}, 5 \%$ urea at $95^{\circ} \mathrm{C}$ for $10 \mathrm{~min}$. After washing in PBS, cells were permeabilized in $0.2 \%$ Triton $\mathrm{X}-100$ for $10 \mathrm{~min}$, and then blocked in PBS containing $0.1 \%$ Tween-20, supplemented with $1 \%$ bovine serum albumin (BSA) and $23 \mathrm{mg} / \mathrm{mL}$ of glycine, for $30 \mathrm{~min}$. Cells were incubated overnight at $4^{\circ} \mathrm{C}$ in $1 \%$ BSA in PBS-T containing 1:50 anti-LC3 antibody (Abcam), and 1:30 anti-P20S (Abcam). Finally, cells were incubated for $1 \mathrm{~h}$ with fluorophore-conjugated secondary antibodies (1:200; goat anti-rabbit Alexa 488 and goat anti-mouse Alexa 594, Molecular Probes, Life Technologies) in $1 \%$ BSA in PBS-T at RT. Cells were washed in PBS, and mounted in ProLong Diamond Antifade Mountant (Molecular Probes, Life Technologies). The analysis was performed by a sequential scanning procedure at Leica TCSSP5 confocal laser scanning micro- 
scope (Leica Microsystems, Mannheim, Germany). Confocal images were collected every $400 \mathrm{~nm}$ intervals through the $z$-axis of each section, through $63 \mathrm{x}$ oil lenses. Z-stacks of serial optical planes were analyzed using the Multicolor Package software.

\section{Chymotrypsin-like P20S enzymatic activity within LC3 immunoprecipitates}

Cells were sonicated at $4{ }^{\circ} \mathrm{C}$ in an ice-cold lysis buffer. One $\mu \mathrm{L}$ of homogenates was used for protein determinations. Proteins ( 800 $\mu \mathrm{g})$ were incubated at $4^{\circ} \mathrm{C}$ overnight with primary rabbit anti-LC3 antibody ( $2 \mu \mathrm{g}$; Sigma-Aldrich, MI, Italy). The antibody/antigen complex was pulled out using protein A-Sepharose beads to isolate LC3-positive precipitates. Chymotrypsin-like enzymatic activity of P20S was measured in these same LC3-immunoprecipitated cell lysates through Chemicon APT280 Proteasome Activity Assay Kit (Merck, Darmstadt, Germany). This is based on the detection of the fluorophore 7-Amino-4-methylcoumarin (AMC) after cleavage from the labeled UP substrate LLVY-AMC. As per manufacturer instructions, after incubation of LC3-immunoprecipitates $(20 \mu \mathrm{L})$ with the kit reagents (final volume $100 \mu \mathrm{L}$ ) for $2 \mathrm{~h}$ at $37^{\circ} \mathrm{C}$, AMC fluorescence was quantified using a $380 / 460 \mathrm{~nm}$ filter set in a $96-$ wells microplate fluorimeter. The protein amount of LC3-positive immunoprecipitates following mTOR inhibition was not normalized on purpose in order to avoid misinterpretation of the assay.
Indeed, the principle of detecting P20S activity within the APP is based on the molecular bond between LC3 and P20S particles. Following rapamycin treatment, there is an increase of LC3 immunoprecipitated fractions, which implies a higher amount of LC3-bound P20S being specifically recruited within APP. Thus, normalizing the amount of LC3 immunoprecipitates following rapamycin would have lowered the amount of LC3 to which PS20 is bound, underestimating P20S activity within the APP.

\section{Statistical analysis}

P20S activity in LC3 immuno-precipitates is expressed as $\mathrm{AMC} \%$ of controls \pm SEM. Statistical analyses to evaluate differences in the P20S enzymatic assay were carried out by using the Student's $t$-test. Null hypothesis (H0) was rejected for $\mathrm{p} \leq 0.05$.

\section{Results and Discussion}

The occurrence of the APP at cellular level was confirmed by confocal microscopy. This is indicated in representative Figure 1 by the merging puncta between LC3 and P20S signals, which is weak at baseline (Figure 1A), and increases impressively following mTOR inhibition by rapamycin (Figure 1B). When analyzed at
A

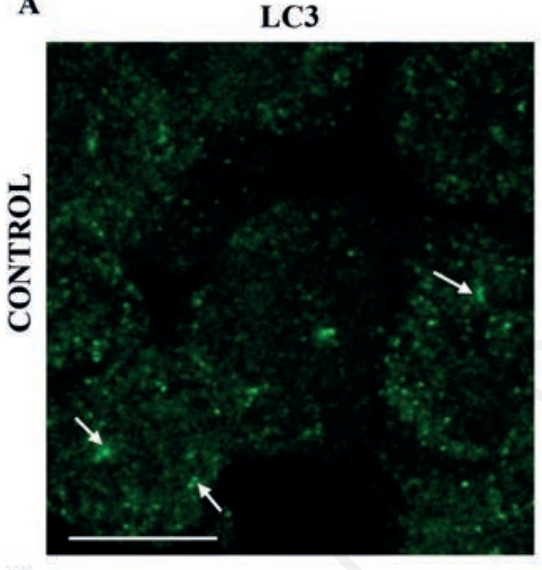

B

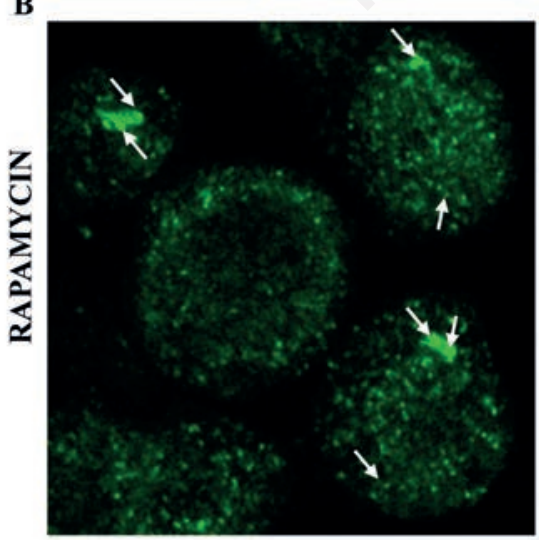

P20S
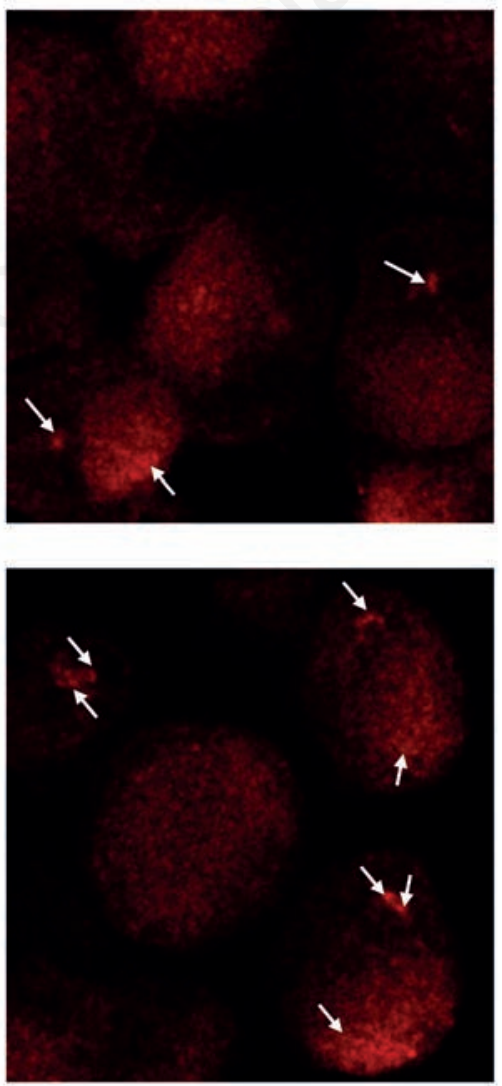

Merge (APP)
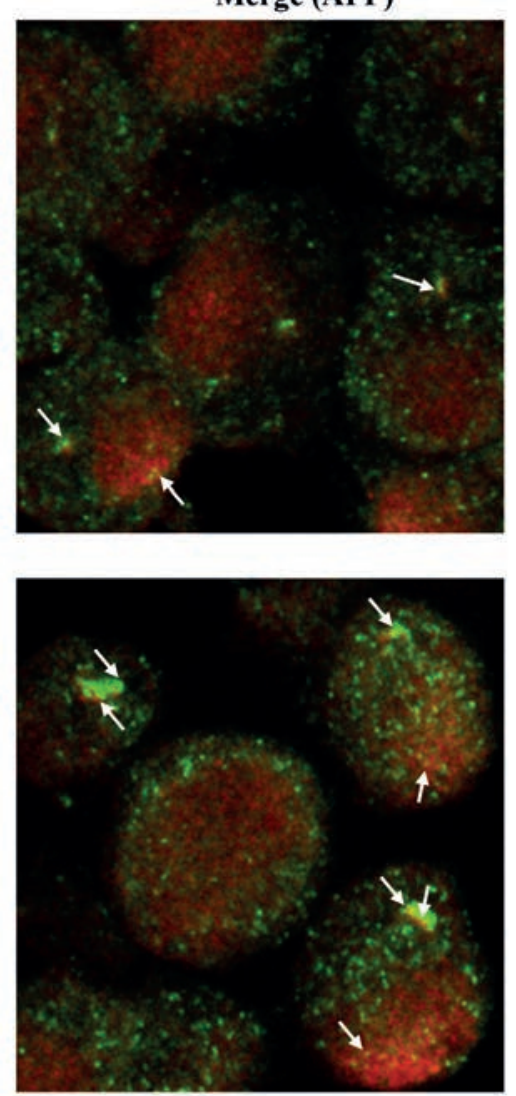

Figure 1. Immunofluorescent detection of APP. Representative immunofluorescence from PC12 cells stained for ATG and UP markers (LC3, green, and P20S red, respectively) and their merging within the APP, at baseline (A) and following mTOR inhibition by rapamycin (B). Arrows point at puncta. Scale bar $=3.5 \mu \mathrm{m}$. 


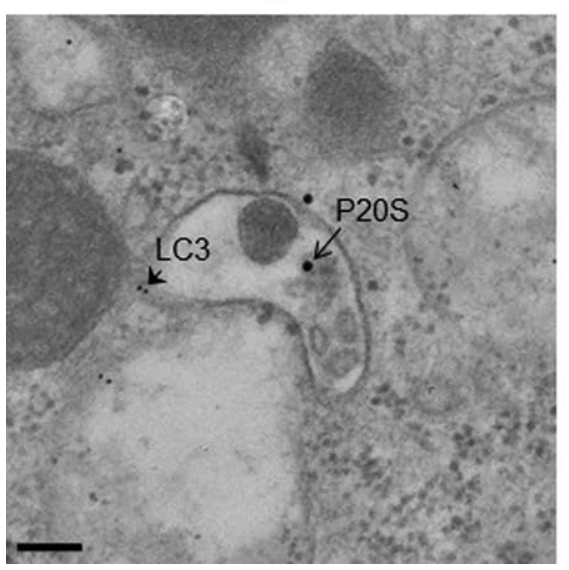

b

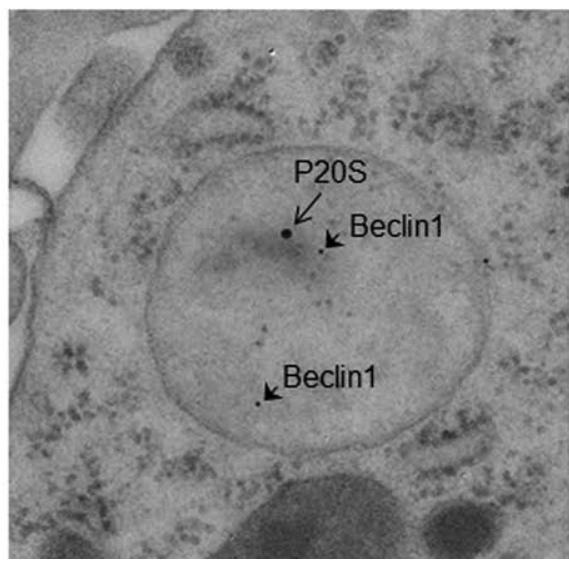

c

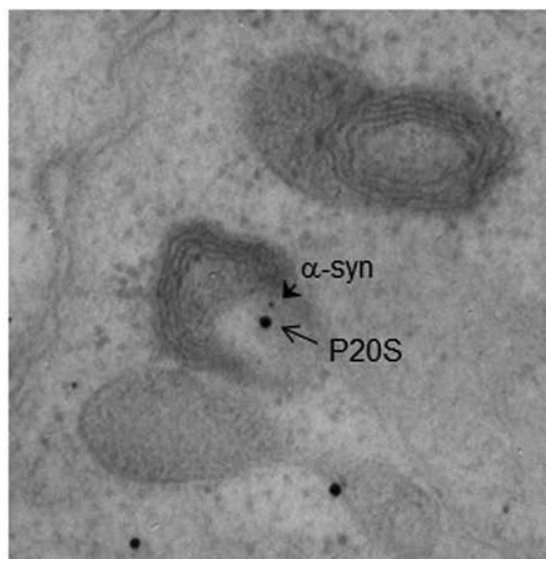

Figure 2. Ultrastructural morphology of APP vacuoles. a) APP staining for LC3 (10 nm, arrowhead) and P20S (20 nm, arrow). This corresponds to a double membrane vacuole at early stages of maturation, as evidenced by the irregular shape and the presence of still undigested vacuolar cargoes. b) APP staining for Beclin1 $(10 \mathrm{~nm}$, arrowhead) and P20S (20 nm, arrow). This corresponds to a mature, gold-standard ATG vacuole in the form of APP, as witnessed by a double membrane, the round shape, the overall electron density which overlaps to that of the cytosol, and the presence of scattered electron dense halos reminiscent of substrates which are going to be degraded. c) Presence of alpha-synuclein $(10 \mathrm{~nm}$, arrowhead) and P20S $(20 \mathrm{~nm}$, arrow) within a multiple membrane APP vacuole at late stages of maturation, as evidenced by the multi-lamellar structures (whorls) which are about to end up in electron dense non-membrane limited bodies. Scale bar=0,3 $\mathrm{nm}$.

ultrastructural morphometry at TEM, these merging units between P20S and LC3 corresponding to APP, appear as vacuoles owning different shape, structure, and electron density according to different maturation stages (Figure 2). In line with our previous studies, and the Autophagy Guidelines ( $4^{\text {th }}$ edition), ${ }^{28,29,33}$ the APP appears as a single-, double-, and multiple-membrane vacuole, where ATG and UP immunogold markers co-localize (Figure 2). In this frame, we extend our previous observations by documenting that beyond LC3, the ATG-related protein Beclin1 is an APP marker as well. Again, ATG vacuoles hosting P20S stain for alpha-synuclein, confirming that misfolded proteins are APP substrates. Finally, preliminary data from UP enzymatic assay measured within LC3immunoprecipitated cell lysates suggest an ongoing UP activity within the APP (Figure 3). Remarkably, the activity of P20S detected at baseline is increased following administration of the mTOR inhibitor rapamycin, confirming at biochemical level our previous morphological evidence on the effects of rapamycin upon APP stimulation. The presence of active UP within APP containing the substrates alpha-synuclein and $\mathrm{p} 62$, coupled with our previous data documenting the neuroprotective effects of APP, ${ }^{29}$ confirms an empowered catalytic activity as the main action of APP, which at late stages may also lead to degradation of UP components (proteaphagy). As described in the latest Autophagy Guidelines (4 $4^{\text {th }}$ edition), the concept of the APP refers to an ultrastructural organelle. ${ }^{33}$ From a functional viewpoint, the APP may feature empowered substrate clearance through the concomitant activity of both ATG and UP, while in other cases the UP component itself may be destroyed under the process of proteaphagy. In our hands, degradation of inactive UP, despite occurring, is not the primary goal of the APP, which indeed empowers vacuolar degradation with strong implications for neuroprotection. Considering the role of ATG and UP in the modulation proteostasis, which is key for a variety of cell functions, these data are expected to provide an experimental platform aimed at dissecting the contribution of such a cell-clearing machinery in physiological and pathological conditions spanning from neurotoxicity up to tumors and neurodegenerative proteinopathies.

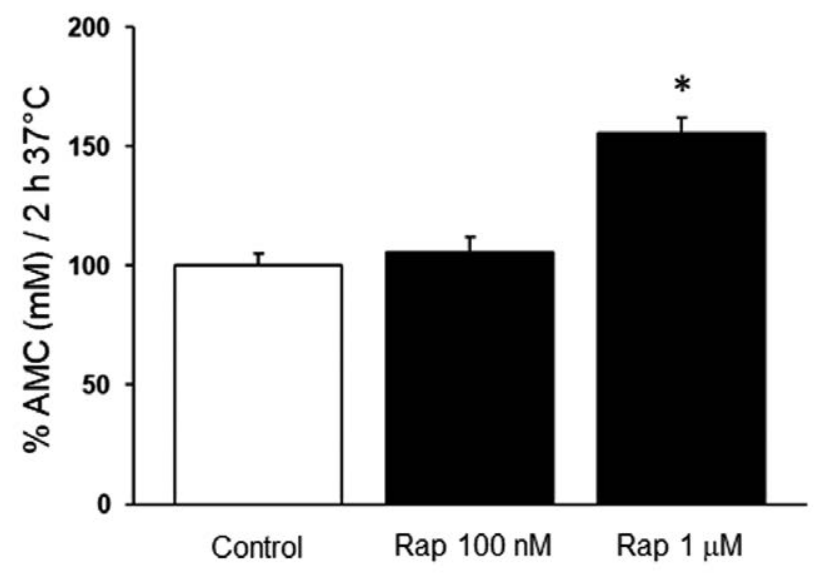

Figure 3. UP enzymatic activity within LC3 immunoprecipitates. Chymotrypsin-like P20S activity is ongoing in baseline conditions, while increases by $50 \%$ following mTOR inhibition by 1 $\mu \mathrm{M}$ rapamycin. P20S activity in LC3 immuno-precipitates is expressed as AMC \% of controls \pm S.E.M. * $\mathrm{p} \leq \mathbf{0 . 0 5}$ vs. control; $\mathrm{RAP}=$ rapamycin . 


\section{References}

1. Klionsky DJ, Emr SD. Autophagy as a regulated pathway of cellular degradation. Science 2000;290:1717-21.

2. Speese SD, Trotta N, Rodesch CK, Aravamudan B, Broadie K. The ubiquitin proteasome system acutely regulates presynaptic protein turnover and synaptic efficacy. Curr Biol 2003;13:899910.

3. Hegde AN. The ubiquitin-proteasome pathway and synaptic plasticity. Learn Mem 2010;17:314-27.

4. Münz C. Autophagy proteins in antigen processing for presentation on MHC molecules. Immunol Rev 2016;272:17-27.

5. Münz C. The macroautophagy machinery in endo- and exocytosis. J Mol Biol 2017;429:473-85.

6. Vijayan V, Verstreken P. Autophagy in the presynaptic compartment in health and disease. J Cell Biol 2017;216:1895906.

7. Limanaqi F, Gambardella S, Biagioni F, Busceti CL, Fornai F. Epigenetic effects induced by methamphetamine and methamphetamine-dependent oxidative stress. Oxid Med Cell Longev 2018;2018:4982453.

8. Limanaqi F, Biagioni F, Gambardella S, Ryskalin L, Fornai F. Interdependency between autophagy and synaptic vesicle trafficking: implications for dopamine release. Front Mol Neurosci 2018,11:299.

9. Limanaqi F, Biagioni F, Busceti CL, Ryskalin L, Fornai F. The effects of proteasome on baseline and methamphetaminedependent dopamine transmission. Neurosci Biobehav Rev 2019;102:308-317.

10. Limanaqi F, Biagioni F, Busceti CL, Ryskalin L, Polzella M, Frati A, et al. Phytochemicals bridging autophagy induction and alpha-synuclein degradation in Parkinsonism. Int $\mathrm{J}$ Mol Sci 2019;20:3274.

11. Limanaqi F, Biagioni F, Gaglione A, Busceti CL, Fornai F. A sentinel in the crosstalk between the nervous and immune system: The (immuno)-proteasome. Front Immunol 2019;10:628.

12. Limanaqi F, Biagioni F, Busceti CL, Ryskalin L, Soldani P, Frati A, et al. Cell clearing systems bridging neuro-immunity and synaptic plasticity. Int J Mol Sci 2019;20:2197.

13. Liang Y. Emerging concepts and functions of autophagy as a regulator of synaptic components and plasticity. Cells 2019;8:34.

14. Limanaqi F, Biagioni F, Gambardella S, Familiari P, Frati A, Fornai F. Promiscuous roles of autophagy and proteasome in neurodegenerative proteinopathies. Int $\mathrm{J}$ Mol Sci 2020;21:3028.

15. Limanaqi F, Busceti CL, Biagioni F, Lazzeri G, Forte M, Schiavon S, et al. Cell clearing systems as targets of polyphenols in viral infections: Potential implications for COVID-19 pathogenesis. Antioxidants (Basel) 2020;9:1105.

16. Fornai F, Lenzi P, Gesi M, Ferrucci M, Lazzeri G, Capobianco $\mathrm{L}$, et al. Similarities between methamphetamine toxicity and proteasome inhibition. Ann N Y Acad Sci 2004;1025:162-70.

17. Fornai F, Schlüter O, Lenzi P, Gesi M, Ruffoli R, Ferrucci M, et al. Parkinson-like syndrome induced by continuous MPTP infusion: Convergent roles of the ubiquitin-proteasome system and alpha-synuclein. Proc Natl Acad Sci USA 2005;102:34138.

18. Fornai F, Lazzeri G, Bandettini Di Poggio A., Soldani P, De Blasi A, Nicoletti F, et al. Convergent roles of alpha-synuclein, DA metabolism, and the ubiquitin-proteasome system in nigrostriatal toxicity. Ann N Y Acad Sci 2006;1074:84-9.

19. Lazzeri G, Lenzi P, Gesi M, Ferrucci M, Fulceri F, Ruggieri S, et al. In PC12 Cells Neurotoxicity Induced by
Methamphetamine Is Related to Proteasome Inhibition. Ann N Y Acad Sci 2006;1074:174-7.

20. Lazzeri G, Lenzi P, Busceti CL, Ferrucci M, Falleni A, Bruno $\mathrm{V}$, et al. Mechanisms involved in the formation of dopamineinduced intracellular bodies within striatal neurons. J Neurochem 2007;101:1414-27.

21. Castino R, Lazzeri G, Lenzi P, Bellio N, Follo C, Ferrucci M, et al. Suppression of autophagy precipitates neuronal cell death following low doses of methamphetamine. J Neurochem 2008;106:1426-39.

22. Mashimoto T, Hadjebi O, Amair-Pinedo F, Tsurumi T, Langa F, Serikawa T, et al. Progressive Purkinje cell degeneration in tambaleante mutant mice is a consequence of a missense mutation in HERC1 E3 ubiquitin ligase. PLoS Genet 2009; 5:e1000784.

23. Friedman LG, Lachenmayer ML, Wang J, He L, Poulose SM, Komatsu M, et al. Disrupted autophagy leads to dopaminergic axon and dendrite degeneration and promotes presynaptic accumulation of $\alpha$-synuclein and LRRK2 in the brain. J Neurosci 2012;32:7585-93.

24. Ruiz R, Pérez-Villegas EM, Bachiller S, Rosa JL, Armengol JA. HERC 1 Ubiquitin Ligase Mutation Affects Neocortical, CA3 Hippocampal and Spinal Cord Projection Neurons: An Ultrastructural Study. Front Neuroanat 2016;10:42.

25. Sato S, Uchihara T, Fukuda T, Noda S, Kondo H, Saiki S, et al. Loss of autophagy in dopaminergic neurons causes Lewy pathology and motor dysfunction in aged mice. Sci Rep 2018;8:2813.

26. Zhao J, Zhai B, Gygi SP, Goldberg AL. mTOR inhibition activates overall protein degradation by the ubiquitin proteasome system as well as by autophagy. Proc Natl Acad Sci USA 2015;112:15790-7.

27. Cohen-Kaplan V, Livneh I, Avni N, Fabre B, Ziv T, Kwon YT, et al. p62-and ubiquitin-dependent stress-induced autophagy of the mammalian 26S proteasome. Proc Natl Acad Sci USA 2016;113:E7490-9.

28. Lenzi P, Lazzeri G, Biagioni F, Busceti CL, Gambardella S, Salvetti A, et al. The autophagoproteasome a novel cell clearing organelle in baseline and stimulated conditions. Front Neuroanat 2016;10:78

29. Lazzeri G, Biagioni F, Fulceri F, Busceti CL, Scavuzzo MC, Ippolito $\mathrm{C}$, et al. mTOR modulates methamphetamine-induced toxicity through cell clearing systems. Oxid Med Cell Longev 2018; 2018:6124745.

30. Seibenhener ML, Babu JR, Geetha T, Wong HC, Krishna NR, Wooten MW. Sequestosome $1 / \mathrm{p} 62$ is a polyubiquitin chain binding protein involved in ubiquitin proteasome degradation. Mol Cell Biol 2004;24:8055-68.

31. Liu WJ, Ye L, Huang WF, Guo LJ, Xu ZG, Wu HL et al. p62 links the autophagy pathway and the ubiqutin-proteasome system upon ubiquitinated protein degradation. Cell Mol Biol Lett 2016;21:29.

32. Otero MG, Alloatti M, Cromberg LE, Almenar-Queralt A, Encalada SE, Pozo Devoto VM, et al. Fast axonal transport of the proteasome complex depends on membrane interaction and molecular motor function. J Cell Sci 2014;127:1537-49.

33. Klionsky DJ, Abdelmohsen K, Abe A, Abedin MJ, Abeliovich $\mathrm{H}$, Acevedo Arozena A, et al. Guidelines for the use and interpretation of assays for monitoring autophagy (4th edition). Autophagy 2021;17:1-382.

34. Marshall RS, Li F, Gemperline DC, Vierstra RD. Autophagic degradation of the $26 \mathrm{~S}$ proteasome is mediated by the dual ATG8/ubiquitin receptor RPN10 in arabidopsis. Mol Cell 2015;58:1053-66.

35. Bendayan M, Zollinger M. Ultrastructural localization of anti- 
genic sites on osmium-fixed tissues applying the protein Agold technique. J Histochem Cytochem 1983;31:101-9.

36. D'Alessandro D, Mattii L, Moscato S, Bernardini N, Segnani $\mathrm{C}$, Dolfi A, et al. Immunohistochemical demonstration of the small GTPase RhoAA on epoxy-resin embedded sections. Micron 2004;35:287-96.

37. Fornai F, Gesi M, Saviozzi M, Lenzi P, Piaggi S, Ferrucci M, et al. Immunohistochemical evidence and ultrastructural compartmentalization of a new antioxidant enzyme in the rat substantia nigra. J Neurocytol 2001;30:97-105.

38. Fornai F, Lenzi P, Gesi M, Soldani P, Ferrucci M, Lazzeri G, et al. Methamphetamine produces neuronal inclusions in the nigrostriatal system and in PC12 cells. J Neurochem 2003;88:114-23. 